\title{
Role of Residual Overturning for the Sensitivity of Southern Ocean Isopycnal Slopes to Changes in Wind Forcing
}

\author{
MADELEINE K. YOUNGS \\ MIT-WHOI Joint Program in Oceanography, Cambridge, Massachusetts \\ GLENN R. FLIERL AND RAFFAELE FERRARI \\ Department of Earth, Atmospheric, and Planetary Sciences, Massachusetts Institute of \\ Technology, Cambridge, Massachusetts
}

(Manuscript received 20 March 2019, in final form 19 August 2019)

\begin{abstract}
The Antarctic Circumpolar Current plays a central role in the ventilation of heat and carbon in the global ocean. In particular, the isopycnal slopes determine where each water mass outcrops and thus how the ocean interacts with the atmosphere. The region-integrated isopycnal slopes have been suggested to be eddy saturated, that is, stay relatively constant as the wind forcing changes, but whether or not the flow is saturated in realistic present day and future parameter regimes is unknown. This study analyzes an idealized two-layer quasigeostrophic channel model forced by a wind stress and a residual overturning generated by a mass flux across the interface between the two layers, with and without a blocking ridge. The sign and strength of the residual overturning set which way the isopycnal slopes change with the wind forcing, leading to an increase in slope with an increase in wind forcing for a positive overturning and a decrease in slope for a negative overturning, following the usual conventions; this behavior is caused by the dominant standing meander weakening as the wind stress weakens causing the isopycnal slopes to become more sensitive to changes in the wind stress and converge with the slopes of a flat-bottomed simulation. Eddy saturation only appears once the wind forcing passes a critical level. These results show that theories for saturation must have both topography and residual overturning in order to be complete and provide a framework for understanding how the isopycnal slopes in the Southern Ocean may change in response to future changes in wind forcing.
\end{abstract}

\section{Introduction}

The Southern Ocean is a dominant contributor to the global carbon and heat budgets, and thus helps determine global climate. The dynamics of the Southern Ocean are dominated by two counterrotating residual overturning cells of about $\sim 10 \mathrm{~Sv}\left(1 \mathrm{~Sv} \equiv 10^{6} \mathrm{~m}^{3} \mathrm{~s}^{-1}\right)$ each (Lumpkin and Speer 2007) forced by surface buoyancy fluxes that transfer tracers from the deep to the surface along isopycnals. In particular, the isopycnal slopes, or equivalently the thermal wind transport, are important for setting the outcropping location of the water masses (with different tracer concentrations) in the Southern Ocean. The isopycnal slopes are also key for determining the depth of the global pycnocline

\footnotetext{
Corresponding author: Madeleine K. Youngs, myoungs@ mit.edu
}

(Gnanadesikan 1999; Nikurashin and Vallis 2011). The Southern Ocean, forced by a strong wind, possesses a strong circumpolar current that is influenced by topography, notably from blocking ridges such as the Scotia Ridge and the Kerguelen Plateau. An analysis of wind data from NCEP and ERA-Interim shows that the maximum zonally averaged wind stress over $1979-2016$ is $0.14 \mathrm{~N} \mathrm{~m}^{-2}$ with a strengthening trend (Lin et al. 2018; Thompson and Solomon 2002; Marshall 2003; Farneti et al. 2015). It is an open question how the Southern Ocean circulation varies with changing wind, and in particular how sensitive the isopycnal slopes are to changing winds with a residual overturning.

In the Southern Ocean, isopycnal slopes are thought to arise from a balance of wind acting to steepen isopycnals and baroclinic eddies acting to flatten them (Johnson and Bryden 1989; Marshall and Radko 2003). Theory suggests a connection between the isopycnal 


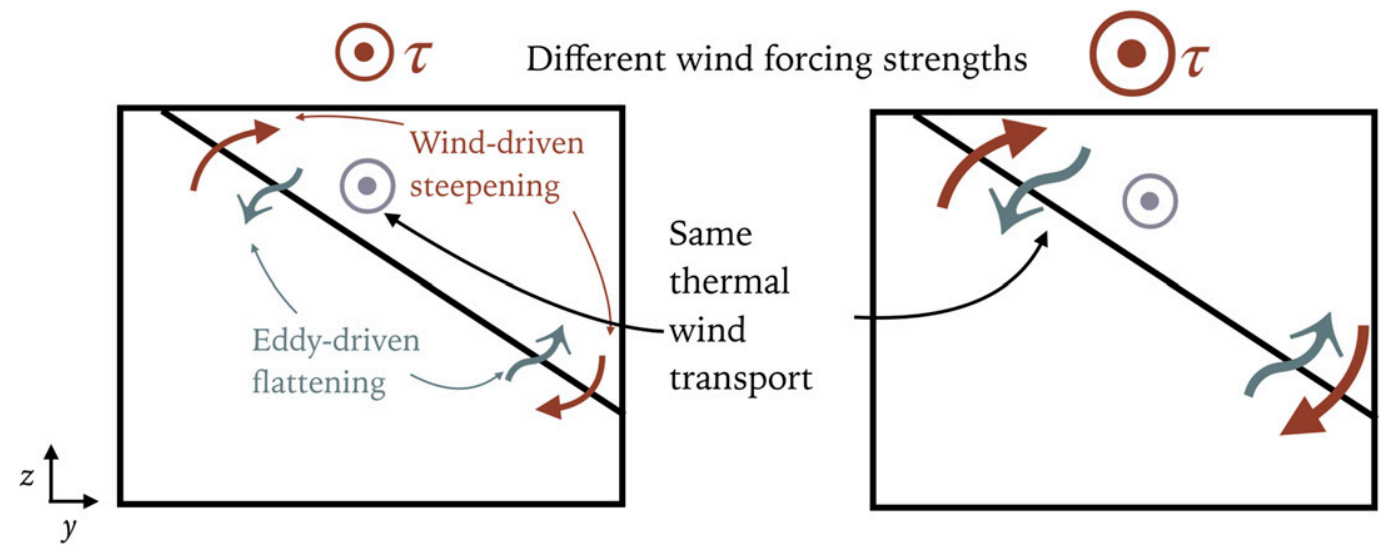

FIG. 1. A schematic describing eddy saturation in the Antarctic Circumpolar Current. When a current is saturated, a change in wind leads to no change in the isopycnal slopes or thermal wind transport.

slopes and the baroclinic eddies, such that, as the wind forcing increases, the eddy fluxes will in kind increase due to increased eddy activity from baroclinic eddies that might be associated with a larger available potential energy reservoir (Danabasoglu et al. 1994; Marshall 1997). This idea has been borne out qualitatively in some observations on short time scales (e.g., Meredith and Hogg 2006; Hogg et al. 2015), in equilibrium in idealized models (e.g., Munday et al. 2013), and on short time scales in a global model (Bishop et al. 2016). This increase in eddy fluxes has led to the hypothesis of "eddy saturation": the isopycnal slopes in the Southern Ocean do not change with increasing wind forcing because eddies become strong enough to counter any increase in wind stress (e.g., Straub 1993; Hallberg and Gnanadesikan 2001) (Fig. 1). Several modeling studies have been found to be consistent with eddy saturation (e.g., Hallberg and Gnanadesikan 2001; Munday et al. 2013; Bishop et al. 2016), but more work is still needed to fully understand under what conditions eddy saturation is realized.

There are several recent hypotheses to explain eddy saturation. One hypothesis suggests that the Southern Ocean is in an equilibrated state where there is an energy balance between eddy energy generation (which depends on isopycnal slopes and eddy energy through an Eady growth rate-like relationship) and the energy decay (which depends on the eddy energy), leading a change in wind stress to increase both the energy generation and decay in equal amounts when certain key parameters, such as drag coefficients, are fixed. The result is a constant transport (Marshall et al. 2017; Mak et al. 2017). Several other theories involve topography. Topography is likely a key aspect of eddy saturation because eddy fluxes tend to peak downstream of major topographic features (e.g., Thompson and Naveira
Garabato 2014; Youngs et al. 2017; Barthel et al. 2017). One hypothesis is that the topography increases the eddy efficiency, so as the wind stress changes the eddy activity changes without the slope needing to change very much to balance the winds (Abernathey and Cessi 2014; MacCready and Rhines 2001). Another theory focuses on the development of gyre circulations downstream of topography; for stronger winds, the barotropic gyres spinup, leaving the thermal wind transport unchanged (Nadeau and Ferrari 2015). Relatedly, Thompson and Naveira Garabato (2014) suggest that it is the flexing of standing meanders downstream of topography that accounts for saturation. Constantinou and Young (2017) and Constantinou (2018) suggest that baroclinic eddies are not even necessary for saturation, invoking a barotropic-topographic instability, albeit in an extreme parameter regime. All of these theories make progress, but do not take into account that there is a residual overturning.

Few studies have connected the Southern Ocean isopycnal slopes and the idea of eddy saturation with the residual overturning present in the system. There have been a couple of papers that examine a Southern Ocean-like system with wind forcing, topography, and a residual overturning in idealized frameworks, highlighting the importance of buoyancy forcing on the isopycnal slopes (e.g., Gnanadesikan and Hallberg 2000; Hallberg and Gnanadesikan 2001; Hogg 2010; Morrison et al. 2011; Shakespeare and Hogg 2012; Howard et al. 2015). Hallberg and Gnanadesikan (2001) use a two-layer primitive equation system with a positive residual overturning forced by an interface relaxation, wind stress, and bottom topography to develop a scaling for the thermal wind transport when the system is dominated by the diabatic forcing, but do not examine the full parameter space, including a lower 


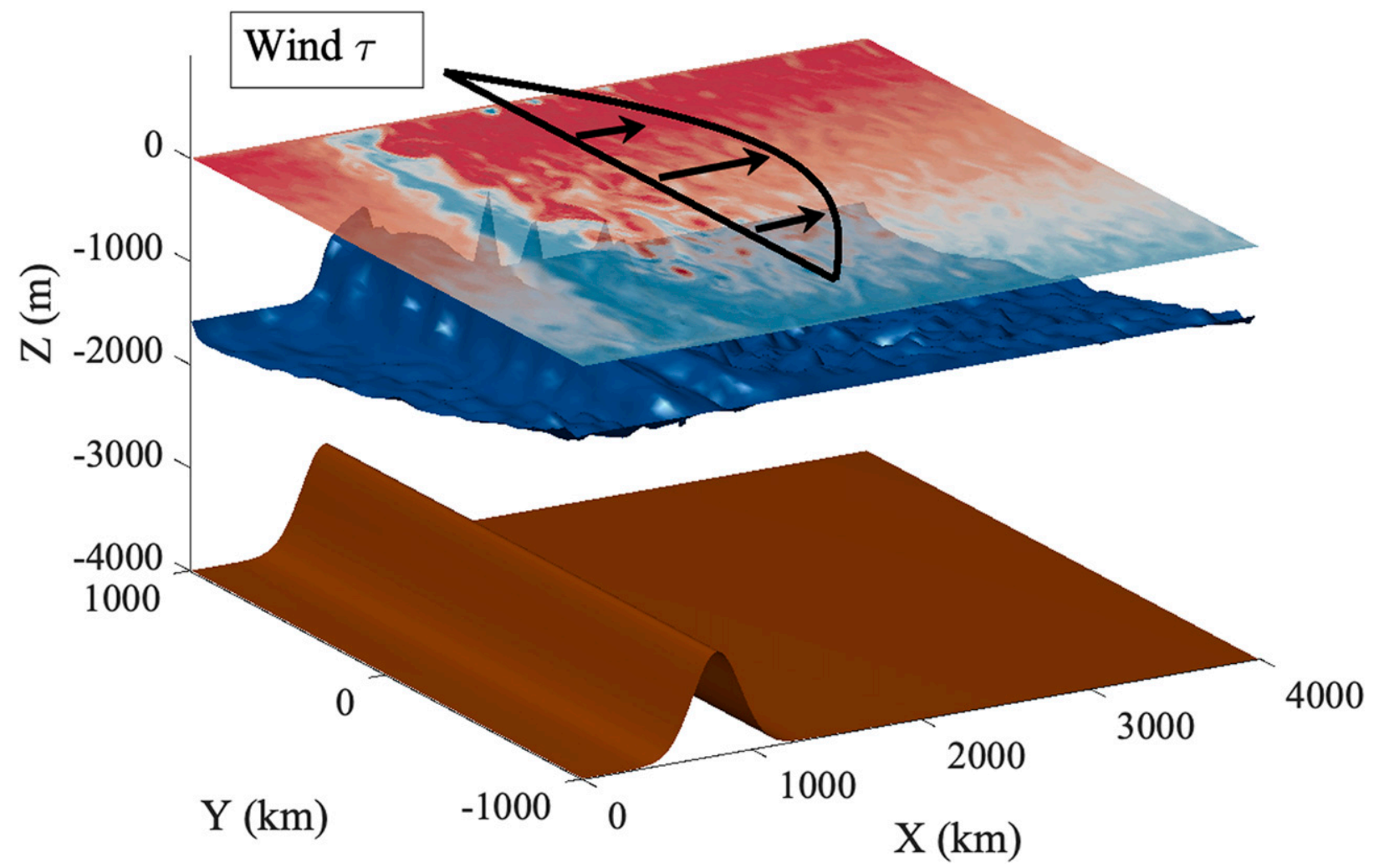

FIG. 2. A three-dimensional figure showing the model domain. The color on the surface is a snapshot of the upper-layer potential vorticity for the case with a wind stress maximum $\tau_{0}=0.2 \mathrm{~N} \mathrm{~m}^{-2}$ and residual overturning $\mathscr{H}_{0}=-2 \mathrm{~Sv}$. The blue surface represents the instantaneous interface height. The wind forcing is shown above the surface.

cell with a negative overturning. Hogg (2010) builds upon Hallberg and Gnanadesikan (2001) to examine a Southern Ocean-like system with a primitive equation model with both buoyancy and wind forcing and find that the system is wind saturated. Howard et al. (2015) examine how the spinup process of a channel differs with wind and buoyancy forcing. Many of these studies have examined only a limited wind- and buoyancy-forcing parameter space, so the validity of eddy saturation over a wide parameter space and the role of buoyancy forcing in modifying how the system responds to winds have not been fully explored.

Here we consider a broad range of forcings to try to gain a more complete picture of the physics of eddy saturation, building upon the work of Hogg (2010) and Howard et al. (2015) to test the theory of saturation with a larger forcing parameter space. We will address how the presence of a residual overturning modifies the response of isopycnal slopes to changing wind forcing.

We use a two-layer quasigeostrophic reentrant channel model with a flat bottom or with a ridge that blocks f/h contours (Fig. 2). The use of simplified models has a long history in being utilized to enhance our understanding of the Southern Ocean (e.g., McWilliams et al. 1978; Hallberg and Gnanadesikan 2001). This particular configuration is a simplified step on the way to understanding the full complexity of the Southern Ocean. At moderate wind stresses, the isopycnal slopes either steepen or flatten with an increasing wind stress, depending on the sign of the residual overturning, until the isopycnal slopes all approach a common value at strong wind stresses. The presence of the residual overturning increases the sensitivity of the isopycnal slopes to changes in wind forcing in the examined range of wind stresses. In section 2, we describe the model configuration and the budgets used for analysis. The results are described in section 3 , and we provide discussions and conclusions in section 4 .

\section{A two-layer quasigeostrophic analog of the Southern Ocean}

\section{a. Model configuration}

The two-layer quasigeostrophic (QG) channel model we use as our analog to the Southern Ocean (Fig. 2) is that described in Flierl and Pedlosky (2007): it solves for the mean zonal flow, the residual meridional overturning 
(combining the ageostrophic mean and the eddy contribution as defined below), and the eddy fields. These elements appear in many previous papers (e.g., Shepherd 1983), however, we present a derivation from the full shallow water, two-layer system in the appendix, showing that the appropriate definition of the residual vector velocity results in very similar equations.

\section{1) MOdel PARAMETERS}

Our channel has free-slip walls at $y= \pm L / 2$ with $L=2000 \mathrm{~km}$ and periodic conditions at $x=0$ and $x=$ $4000 \mathrm{~km}$ (Table 1). The upper layer has thickness $h_{1}=$ $H_{1}+h$ with the mean value $H_{1}=2 / 3 \mathrm{~km}$; the lower layer has $h_{2}=H_{2}-h-h_{b}(x)$ and $H_{2}=3^{1 / 3} \mathrm{~km}$. We picked the relative layer thicknesses based on the stratification as observed in the Southern Ocean (Karsten and Marshall 2002). The reduced gravity $g^{\prime}$ is chosen such that the deformation radius is $15 \mathrm{~km}$, based on an estimation of the value for much of the Southern Ocean (Chelton et al. 1998) and a reference density $\rho_{0}=$ $1000 \mathrm{~kg} \mathrm{~m}^{-3}$, used later for scaling. The grid size is around $15 \mathrm{~km}$, and, even though it is around the same size as the deformation radius $r_{d}$, we expect the dynamics will be adequately resolved since the wavelength of the fastest growing baroclinic instability wave is order $10 r_{d}$ and the nonlinear motions tend to be several times larger than $r_{d}$ (Pedlosky 1987). Additionally, we have run several key simulations at twice the resolution that lead to the exact same conclusions concerning the response of the thermal wind transport to the winds and less than a $10 \%$ difference in the thermal wind transport. Nonconservative terms are wind stress $\tau$, a linear bottom drag with coefficient $\mu$, and buoyancy forcing $\mathscr{H}$, which represents warming that transforms lower-layer water to upper-layer water or cooling that does the opposite. We also include a small downgradient potential vorticity flux with diffusivity $\kappa$ to remove small-scale enstrophy. See Table 1 for parameter values.

\section{2) TOPOGRAPHY}

The elevation of the topographic ridge $h_{b}(x)$ varies only zonally. We have performed simulations with two different topographies, a flat bottom $h_{b}=0 \mathrm{~m}$ and a simple cross channel Gaussian ridge centered at $x=x_{0}$ :

$$
h_{b}(x)=h_{0} e^{-\left(x-x_{0}\right)^{2} / \sigma^{2}},
$$

where $h_{0}=1000 \mathrm{~m}, \sigma=200 \mathrm{~km}$, and $x_{0}=800 \mathrm{~km}$. The total depth away from the topography is $4000 \mathrm{~m}$, and the ridge is $30 \%$ of the lower layer thickness. We also ran simulations with $h_{0}=2000 \mathrm{~m}$ for verification and find that the thermal wind transport saturates to the
TABLE 1. Table showing various parameters used for two-layer quasigeostrophic model.

\begin{tabular}{clc}
\hline \hline Parameter & \multicolumn{1}{c}{ Description } & Value \\
\hline$\mu$ & Bottom drag & $3.47 \times 10^{-7} \mathrm{~s}^{-1}$ \\
$r_{d}$ & Deformation radius & $15 \mathrm{~km}$ \\
$H_{1}$ & Upper-layer thickness & $2 / 3 \mathrm{~km}$ \\
$H_{2}$ & Lower-layer thickness & $31 / 3 \mathrm{~km}$ \\
$\kappa$ & Potential vorticity diffusivity & $15 \mathrm{~m}^{2} \mathrm{~s}^{-1}$ \\
$f_{0}$ & Coriolis frequency & $-1 \times 10^{-4} \mathrm{~s}^{-1}$ \\
$\beta$ & Meridional derivative of Coriolis & $1 \times 10^{-11} \mathrm{~m}^{-1} \mathrm{~s}^{-1}$ \\
& frequency & $15.625 \mathrm{~km}^{-3}$ \\
$\rho_{0}$ & Grid size & $1000 \mathrm{~kg} \mathrm{~m}^{-3}$ \\
\hline
\end{tabular}

same value but reaches the saturation at a smaller wind stress. However, we focus on $h_{0}=1000 \mathrm{~m}$ because the smaller ridge is more consistent with the quasigeostrophic assumptions.

\section{3) RESIDUAL FORMULATION}

To recast the mass equation in our residual flow formulation, we split the mass transport within a layer into a rotational and divergent part (Kushner and Held 1999):

$$
\mathbf{u}_{i}^{*}=\mathbf{u}_{i} \frac{h_{i}}{H_{i}}-\hat{\mathbf{z}} \times \nabla \psi_{i} \Rightarrow \nabla \cdot\left(\mathbf{u}_{i} h_{i}\right)=H_{i} \nabla \cdot \mathbf{u}_{i}^{*},
$$

where $\psi_{i}$ is the rotational component of the horizontal velocities $\mathbf{u}, \nabla$ represents a horizontal gradient, and ()* represents the residual component. The zonally averaged mass equation becomes linear:

$$
\begin{gathered}
\frac{\partial}{\partial t}\left\langle h_{1}\right\rangle+\frac{\partial}{\partial y}\left\langle h_{1} v_{1}\right\rangle=\frac{\partial}{\partial t}\left\langle h_{1}\right\rangle+H_{1} \frac{\partial}{\partial y}\left\langle v_{1}^{*}\right\rangle=\langle\mathscr{H}\rangle \\
\text { or } \quad \frac{\partial}{\partial t}\left\langle h_{1}\right\rangle+\left\langle w^{*}\right\rangle=\langle\mathscr{H}\rangle,
\end{gathered}
$$

where the angle brackets denote a zonal average. The residual vertical velocity $w^{*}=w^{a}+\mathbf{u}_{1} \cdot \nabla h_{1}$ relates the cross-interface flow to the two components of the up- or downslope velocity, with $w^{a}$ being the actual vertical velocity including that associated with alongslope motions (with the superscript emphasizing that the velocity is ageostrophic). Equation (1) shows not only the link between the residual overturning $H_{1}\left\langle v^{*}\right\rangle=$ $\left\langle h_{1} v_{1}\right\rangle$ and the buoyancy forcing but also the way in which the residual flow encompasses both the ageostrophic and eddy fluxes, with the two on average cancelling when $\mathscr{H}=0$ and, in general, partially offsetting each other. Note that the displacement of the top surface can be neglected in the mass equation so that $\partial h_{2} / \partial t=-\partial h_{1} / \partial t$; this implies that $H_{1}\left\langle v_{1}^{*}\right\rangle+$ $H_{2}\left\langle v_{2}^{*}\right\rangle=0$, and we only need to find the residual circulation in the upper layer. 


\section{4) EQUATIONS OF MOTION}

As detailed in the appendix, using the definitions above for $\mathbf{u}_{i}^{*}$ and $w^{*}$, supplemented with $p_{i}^{*}=p_{i}-f_{0} \psi_{i}+$ $\left|\mathbf{u}_{i}\right|^{2} / 2$, and then making the usual small Rossby number expansion leads to the quasigeostrophic equations in the two layers (Pedlosky 1987):

$$
\frac{\partial}{\partial t} q_{i}+\mathbf{u}_{i} \cdot \nabla q_{i}= \pm f_{0} \frac{\mathscr{C}}{H_{i}}-\delta_{i 1} \frac{\tau_{y}}{\rho_{0} H_{1}}+\kappa \nabla^{2} q_{i}-\delta_{i 2} \mu \nabla^{2} \psi_{i} .
$$

The potential vorticity is $q_{i}=f_{0}+\beta y+\delta_{i 2} f_{0} h_{b} / H_{2}+$ $\nabla^{2} \psi_{i} \mp F_{i}\left(\psi_{1}-\psi_{2}\right)$, and $F_{i}=f_{0}^{2} / g^{\prime} H_{i}$ are the stratification parameters, which are related to the deformation radius $r_{d}$ by $F_{1}+F_{2}=1 / r_{d}^{2}$. We have included a small diffusivity $\kappa$ that is generally negligible unless there are no eddies to transport energy vertically downward where it can be dissipated by bottom drag.

It is useful both computationally and for diagnostics to split out the zonal mean flow and advance $\left\langle u_{i}\right\rangle$ with the zonally averaged momentum equation

$$
\frac{\partial}{\partial t}\left\langle u_{i}\right\rangle-f_{0}\left\langle v_{i}^{*}\right\rangle-\left\langle v_{i}^{\prime} q_{i}^{\prime}\right\rangle=\delta_{i 1} \frac{\tau}{\rho_{0} H_{1}}-\delta_{i 2} \mu\left\langle u_{2}\right\rangle,
$$

where primes represent a deviation from the zonal average. We can use the thermal wind equation,

$$
f_{0}\left(\left\langle u_{1}\right\rangle-\left\langle u_{2}\right\rangle\right)=g^{\prime} \partial h_{1} / \partial y
$$

to find a diagnostic equation for $\left\langle v_{1}^{*}\right\rangle$

$$
\begin{aligned}
\left(\frac{\partial^{2}}{\partial y^{2}}-F_{1}-F_{2}\right)\left\langle v_{1}^{*}\right\rangle= & \frac{f_{0}}{g^{\prime} H_{1}}\left(\left\langle v_{i}^{\prime} q_{1}^{\prime}\right\rangle-\left\langle v_{2}^{\prime} q_{2}^{\prime}\right\rangle+\frac{\tau}{\rho_{0} H_{1}}\right. \\
& \left.+\mu\left\langle u_{2}\right\rangle\right)+\frac{1}{H_{1}} \frac{\partial}{\partial y}\langle\mathscr{B}\rangle .
\end{aligned}
$$

The zonally varying QG equations

$$
\frac{\partial}{\partial t} q_{i}^{\prime}+\left\langle u_{i}\right\rangle \frac{\partial}{\partial x} q_{i}^{\prime}+\nabla \cdot\left(\mathbf{u}_{i}^{\prime} q_{i}^{\prime}\right)-\frac{\partial}{\partial y}\left\langle v_{i}^{\prime} q_{i}^{\prime}\right\rangle=\kappa \nabla^{2} q_{i}-\delta_{i 2} \mu \nabla^{2} \psi_{i}
$$

together with Eqs. (3) and (5) comprise our model equations.

\section{5) MODEL FORCING}

The buoyancy forcing - cross-isopycnal mass transferis taken to be $\mathscr{H}=-\mathscr{H}_{0} \sin (\pi y / L)$. To put $\mathscr{H}_{0}$ in perspective, consider the upper-layer mass equation

$$
\frac{\partial}{\partial t} h_{1}+\nabla \cdot\left(\mathbf{u}_{1} h_{1}\right)=\mathscr{H},
$$

which takes the form

$$
\frac{\partial}{\partial t}\left\langle h_{1}\right\rangle+\frac{\partial}{\partial y}\left\langle v_{1} h_{1}\right\rangle=\mathscr{B}
$$

after a zonal mean (indicated by angle brackets). Thus, the time-mean northward mass transport-the meridional overturning —is just

$$
\left\langle v_{1} h_{1}\right\rangle \equiv\left\langle v_{1}^{a}\right\rangle\left\langle h_{1}\right\rangle+\left\langle v_{1}^{\prime} h_{1}^{\prime}\right\rangle=\langle\mathscr{H}\rangle=H_{1}\left\langle v_{1}^{*}\right\rangle,
$$

with $v^{a}$ the ageostrophic velocity (since the geostrophic northward velocity has zero zonal average). We choose $\mathscr{H}$ to drive a northward transport - the residual overturning - of 2 or $4 \mathrm{~Sv}$ because our channel is about 1/4 the length of the Antarctic Circumpolar Current. Our cases would represent 8 and $16 \mathrm{~Sv}$ of overturning with a channel the length of the Antarctic Circumpolar Current (ACC) compared to the estimate of $10 \mathrm{~Sv}$ in the Southern Ocean. ${ }^{1}$ When we discuss positive overturning we mean that $\mathscr{H}_{0}>0$ and time-averaged $\left\langle h_{1} v_{1}\right\rangle>0$, so that there is sinking in the north and upwelling in the south; negative overturning has the opposite sense with upwelling in the north and sinking in the south.

We want to explore a range of wind and buoyancy forcings but identify those we consider to be "realistic" to assess the sensitivity of the isopycnal slopes to changes in forcing within a range that might conceivably apply to Southern Ocean. Given the idealizations of the model, we cannot expect precise matching. We assume wind stresses of the form

$$
\tau=\tau_{0} \cos (\pi y / L)
$$

and vary $\tau_{0}$ between 0 and $0.8 \mathrm{~N} \mathrm{~m}^{-2}$. Observations (Lin et al. 2018; Farneti et al. 2015) suggest $\tau_{0}$ is about $0.14 \mathrm{~N} \mathrm{~m}^{-2}$, so we will consider $\tau_{0}>0.2 \mathrm{~N} \mathrm{~m}^{-2}$ to be "strong" and less than $0.1 \mathrm{Nm}^{-2}$ "weak." To put these in a similar context to the buoyancy forcing, we look at the mean zonal momentum equations [Eq. (3)]. If we multiply the upper layer equation by $H_{1} / f_{0}$, we can then compare the residual overturning transport to the eddy transport and wind-driven overturning. The scaled wind stress term is just the Ekman transport $\tau / \rho_{0} f_{0} ; \tau_{0}=$ $0.1 \mathrm{~N} \mathrm{~m}^{-2}$ would generate a $4 \mathrm{~Sv}$ transport, smaller values than this indicate weak wind transports. Ekman transports larger than $8 \mathrm{~Sv}$ would indicate large wind transports. Of course, in the absence of buoyancy forcing,

\footnotetext{
${ }^{1}$ Since the ACC traverses multiple topographic features, modeling a segment of it rather than a single ridge in a longer channel seems appropriate.
} 

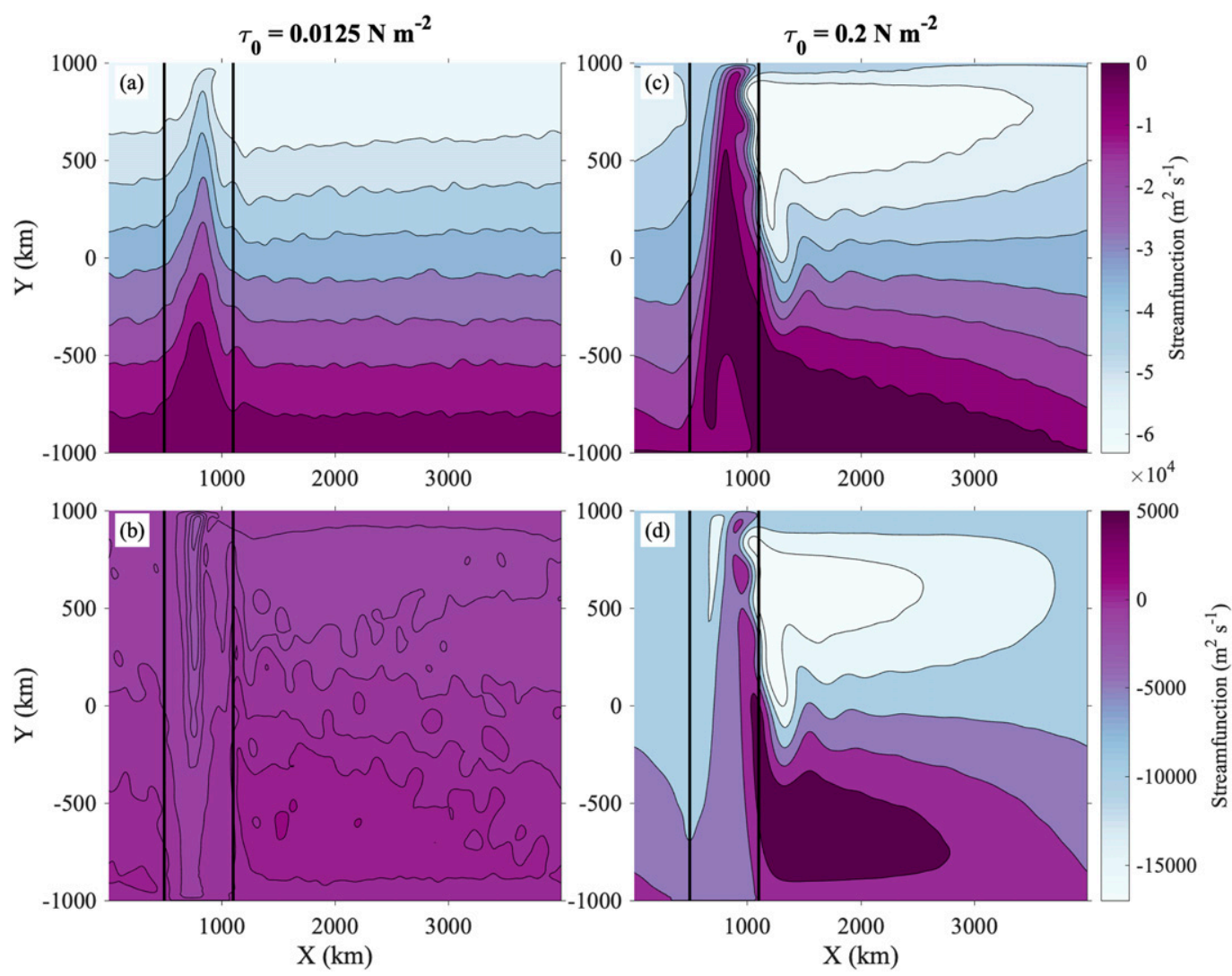

FIG. 3. The streamfunctions for the cases where residual overturning $\mathscr{H}_{0}=-2 \mathrm{~Sv}$ for (a),(b) weak winds of $\tau_{0}=$ $0.0125 \mathrm{~N} \mathrm{~m}^{-2}$ and (c),(d) relatively strong winds of $\tau_{0}=0.2 \mathrm{~N} \mathrm{~m}^{-2}$. The time averaged upper-layer transport $\overline{\psi_{1}}$ is shown in (a) and (c); the time averaged lower-layer transport $\overline{\psi_{2}}$ is shown in (b) and (d). The black lines indicate where the topography exceeds $100 \mathrm{~m}$.

this transport would be on average balanced by an opposite eddy mass flux. We note that wind stress curl is an important part of subduction and therefore meridional overturning in a fully stratified ocean (Marshall and Speer 2012); in our quasigeostrophic setup, the "subduction" is fixed even though the wind stress curl is proportional to $\tau_{0}$.

\section{6) THERMAL WIND TRANSPORT}

We wish to diagnose the mean isopycnal slopes, so we utilize the relationship between the isopycnal slopes and the transport through the thermal wind relationship created by combining Eqs. (1) and (3). We calculate the thermal wind transport

$$
T_{w}=H_{1} \int\left(\left\langle u_{1}\right\rangle-\left\langle u_{2}\right\rangle\right) d y,
$$

that is, the two-layer analog of the transport calculated geostrophically, assuming a level of motion at the bottom, to highlight the connection between the transport and the isopycnal slopes. "Baroclinic transport" is often used for this quantity, but we prefer not to use this term since it is confusing when thinking in terms of vertical modes.

\section{7) IMPORTANT LIMITS}

For almost the entire range of wind stresses and residual overturnings that we test, the system is vigorously eddying; the simulations with a ridge also exhibit a large standing eddy or gyre downstream of the ridge, coincident with the most strongly eddying flow (Fig. 3). For a flat-bottomed situation, the lower-layer potential vorticity gradient changes sign when the thermal wind transport reaches $18 \mathrm{~Sv}$, rendering the system susceptible to baroclinic instability. For the linear problem, we can calculate the forcing that first generates eddies. If there is just a residual overturning, that value is about $\mathscr{H}_{0}=-0.03 \mathrm{~Sv}$. If there is only a wind stress, a forcing of about $\tau_{0}=10^{-3} \mathrm{~N} \mathrm{~m}^{-2}$ is sufficient to reverse the potential vorticity gradients. These particular values depend on potential vorticity diffusivity $\kappa$. We do not consider positive residual overturning and wind forcing combinations that have westward transport: the gradient of the Coriolis parameter $\beta$ imposes 


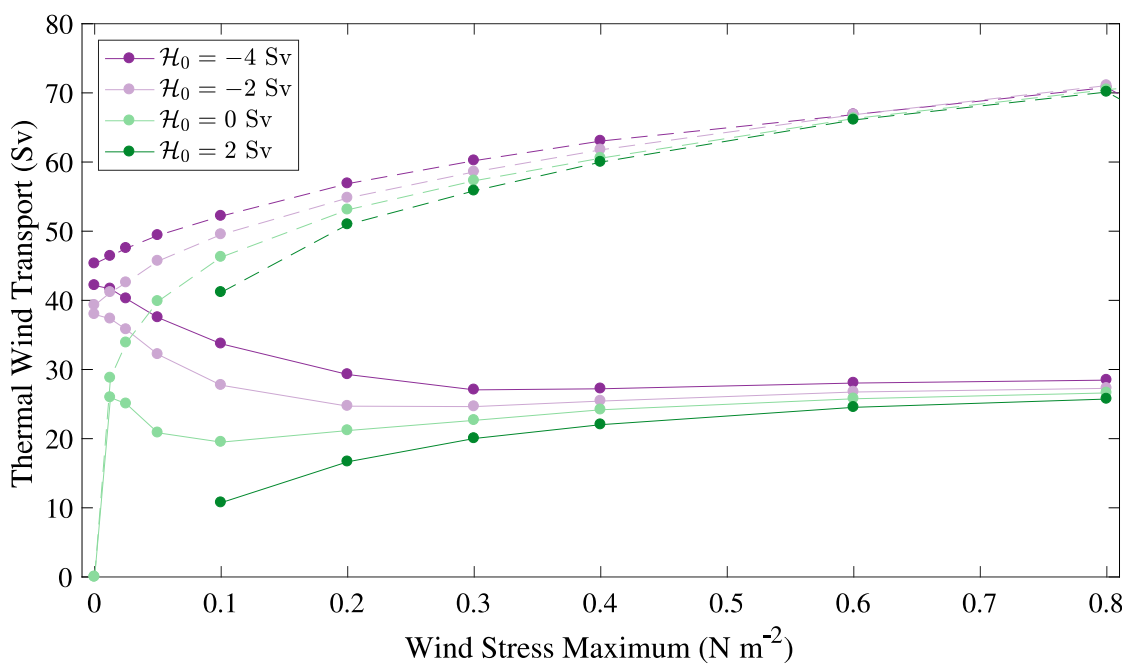

FIG. 4. Thermal wind transport vs wind stress for four different overturnings with (solid) and without (dashed) a 1000-m-tall ridge. At strong wind stresses there is saturation, but as the wind forcing weakens to moderate, present-day levels, the slopes change with the wind, in different directions depending on the sign of the overturning in order to approach the transport for a flat bottom. When the wind stress is very small and the overturning positive, the flow is westward and will not be discussed further.

an asymmetry between eastward and westward flow, and hence a westward flow behaves very differently and puts itself outside the parameter space that is relevant to the Southern Ocean.

\section{b. Connection between overturning and slope}

The common conceptual ideas of saturation usually involve steepening of the thermocline slope by increased wind being offset by baroclinic instability fluxing heat and acting to flatten the thermocline slope, making the thermal wind transport relatively insensitive to the strength of the wind forcing. However, it turns out that topography plays a crucial role in saturation, for example, by modifying the baroclinic activity (Abernathey and Cessi 2014). Given the significant changes in the flow structure associated with topography or heat flux, it seems important to examine how the transport varies with an interface heat flux as well. The addition of a heat flux across the interface certainly modifies the isopycnal slope.

We will examine how in the following thought experiment. Consider the following: start with a system with no buoyancy forcing in equilibrium and consider the time-averaged circulations. The residual velocity is zero [Eq. (1)] and the upper-layer momentum equation [Eq. (3)] implies that the wind stress is balanced by southward eddy potential vorticity fluxes. With eastward upper-layer flow $\left\langle u_{1}\right\rangle$, we expect from the thermal wind equation that the interface will be shallow at the south and deep at the north, that is, $g^{\prime} \partial\langle h\rangle / \partial y \sim-f_{0}\left\langle u_{1}\right\rangle>0$. When we add a buoyancy flux $\mathscr{H}_{0}<0$ such that there is cooling in the south and warming in the north, the interface will begin to tilt further. This is partially impeded by a southward residual flux $\left(\partial\left\langle v_{1}^{*}\right\rangle / \partial y\right.$, which is negative in the south and positive in the north). Equation (3) then shows that the eddy potential vorticity flux must become more negative to halt the steepening of the interface. This is consistent with enhanced baroclinic instability. But we are left with a net increase in the interface slope and the thermal-wind transport when the overturning is negative. Likewise, with a positive residual overturning $\left(\mathscr{H}_{0}>0\right)$, the interface slope will decrease compared to when there is no overturning (Fig. 4).

\section{c. Budgets}

Splitting out the zonal average provides as a useful framework for physical interpretation. The time average (indicated by an overbar) of the upper-layer momentum equation [Eq. (3)], taking advantage of $\tau$ depending only on $y$ results in

$$
\left\langle\overline{v_{1}^{\prime} q_{1}^{\prime}}\right\rangle+f_{0}\left\langle\overline{v_{1}^{*}}\right\rangle+\frac{\tau}{\rho_{0} H_{1}}=0
$$

and expresses the trade-off between wind stress, residual overturning, and eddy fluxes in increasing (if positive) or decreasing (if negative) the upper layer zonal flow. Residual overturning from the time-mean mass equation [Eq. (1)] 


$$
H_{1} \frac{\partial}{\partial y}\left\langle\overline{v_{1}^{*}}\right\rangle=\mathscr{H}
$$

can be integrated in $y$ and yields our momentum balance diagnostic:

$$
\left\langle\overline{v_{1}^{\prime} q_{1}^{\prime}}\right\rangle+\frac{f_{0}}{H_{1}} \int_{0}^{y} \mathscr{H}\left(y^{\prime}\right) d y^{\prime}+\frac{\tau}{\rho_{0} H_{1}}=0,
$$

or, for the specific forms of $\tau$ and $\mathscr{H}$ used,

$$
\left\langle\overline{v_{1}^{\prime} q_{1}^{\prime}}\right\rangle=-\frac{f_{0}}{H_{1}}\left(\frac{L}{\pi} \mathscr{H}_{0}+\frac{\tau_{0}}{\rho_{0} f_{0}}\right) \cos (\pi y / L) .
$$

For $\tau_{0}>0$ and $f_{0}<0$, the eddy potential vorticity flux must be stronger (weaker) for negative (positive) overturning.

The upper-layer potential vorticity flux has a relative vorticity contribution $\left\langle\overline{\left\langle v_{1}^{\prime} \nabla^{2} \psi_{1}^{\prime}\right.}\right\rangle$ and a thickness flux $-f_{0} / H_{1}\left\langle\overline{v_{1}^{\prime} h^{\prime}}\right\rangle$. If we neglect the former, since it has no contribution in the domain integral and is generally small in our experiments, then the eddy thickness (or heat) flux is

$$
\left\langle\overline{\boldsymbol{v}_{1}^{\prime} h^{\prime}}\right\rangle=\left(\mathscr{H}_{0}+\frac{\tau_{0}}{\rho_{0} f_{0}}\right) \cos (\pi y / L)
$$

Since the derivation of Eq. (10) also uses the thickness and heat equations, we could instead think about this equation as a heat or thickness budget (Plumb and Ferrari 2005). In this heat budget, the wind stress term represents the mean advection of heat by the ageostrophic flow, and the eddy terms are the eddy advection of heat, and the interface forcing is the input of heat. Similarly, this balance could also be thought of as the different components of the residual overturning, where the total residual overturning given by the interface forcing is just the balance between the wind-driven overturning and eddy-driven overturning.

Summing the upper- and lower-layer momentum equations, weighted by the layer thicknesses equations, gives the traditional momentum balance:

$$
\frac{\tau}{\rho_{0}}=f_{0}\left\langle h_{b} \bar{\psi}_{2 x}^{\prime}\right\rangle+\mu H_{2}\left\langle u_{2}\right\rangle,
$$

where the subscript $x$ indicates a derivative with respect to $x$. For the simulations with a ridge, the form drag $\left(f_{0}\left\langle h_{b} \bar{\psi}_{2 x}^{\prime}\right\rangle\right)$, from the difference in geostrophic pressure on either side of the topography, balances the momentum input, whereas for the flat bottom case, the momentum input by the wind is removed by bottom drag (Munk and Palmén 1951).

\section{Results}

We investigate two main questions: where in the parameter space is the flow saturated, and what role does the residual overturning play in setting the thermal wind transport? The simulations performed have several different residual overturnings over a range of wind stresses (Fig. 2). We analyze the resulting thermal wind transport, heat fluxes, and form drag in order to understand how and why the isopycnal slopes vary over the wide range of forcings examined.

\section{a. Strong wind forcing}

There is a history of studies that find saturation in a channel with a blocking ridge and no saturation with a flat bottom (e.g., Munday et al. 2015; Nadeau and Ferrari 2015). Indeed, we see in Fig. 4 that the thermal wind transport still changes with wind stress in the flat-bottomed simulations across the imposed wind stresses. By comparison, we see eddy saturation in the simulations with a ridge, but only at strong wind forcing, $\tau_{0}>0.2 \mathrm{~N} \mathrm{~m}^{-2}$. On the other hand, the vertically integrated transport is essentially constant for wind stress $\tau_{0}<0.2 \mathrm{Nm}^{-2}$, but increases linearly with the wind stress when $\tau_{0}>0.2 \mathrm{~N} \mathrm{~m}^{-2}$ (not shown).

Since we see that the flow is saturated at strong wind forcing, we question what allows eddy saturation. We calculate the eddy heat fluxes from Eq. (10) and normalize them by dividing by the total heat transport. We see that the normalized heat fluxes are concentrated into the meander regions when the wind forcing is strong and that in the downstream region the heat fluxes are irrelevant (Fig. 5). This result has also been seen in MacCready and Rhines (2001) and Abernathey and Cessi (2014). The meander strengthens the temperature gradients locally, leading to larger heat fluxes across mean streamlines. However, the gradients tend to be zonal (and opposite signed on either side of the ridge) instead of meridional, leading to very little change in the zonally averaged isopycnal slopes. Overall, the total heat fluxes are increasing linearly with the wind, but are localized near the ridge, indicating that it is primarily the meander that is contributing to all of the changes in eddy fluxes. Thus, understanding the meander system is incredibly important for understanding the saturation process.

\section{b. Realistic winds}

Outside of the strong wind forcing limit, that is, for $\tau_{0}<0.2 \mathrm{~N} \mathrm{~m}^{-2}$, the thermal wind transport is more sensitive to changes in wind forcing (Fig. 4). In the Southern Ocean, a realistic mean wind stress range is about $0.1-0.2 \mathrm{~N} \mathrm{~m}^{-2}$ ( $\mathrm{Lin}$ et al. 2018). In this range, 

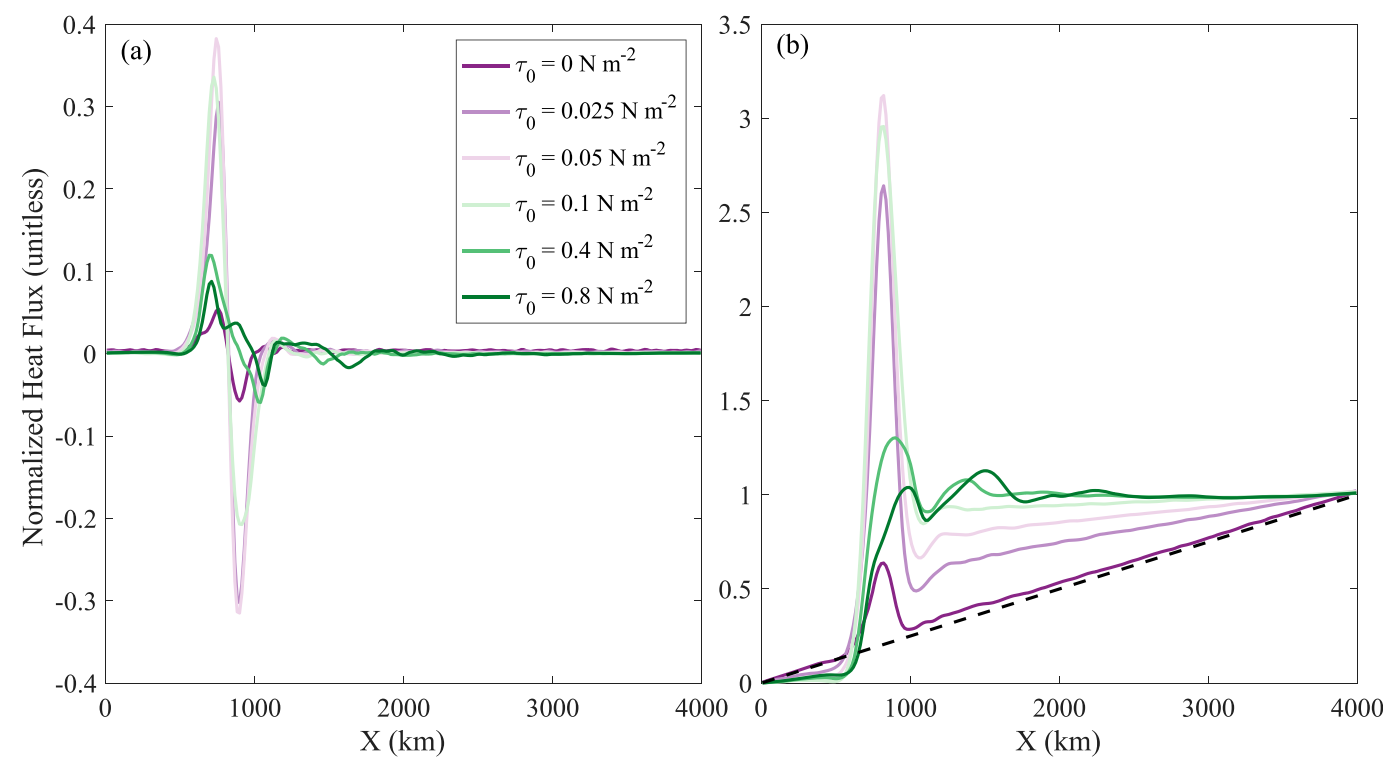

FIG. 5 (a) Eddy heat fluxes normalized by the total heat fluxes along the channel for $\mathscr{H}_{0}=-2 \mathrm{~Sv}$. Most of the heat fluxes occur in or just downstream of the meander. (b) The cumulative normalized eddy heat flux along $x$, indicating that when the wind is strong, most of the heat fluxes occur in the meander, but with weaker winds, the heat fluxes throughout the whole domain are just as important. The black dashed line shows the heat fluxes for a flat-bottomed experiment.

thermal wind transport either increases or decreases with the wind stress, depending on the sign of the overturning, so we see that saturation breaks down in our idealized model at "realistic" wind stresses.

So what is happening as the wind forcing gets weaker? The dominant momentum balance is given by Eq. (11), or, to lowest order,

$$
\frac{\tau}{\rho_{0}}=f_{0}\left\langle h_{b} \bar{\psi}_{2 x}^{\prime}\right\rangle
$$

while $\tau_{0}>0.0125 \mathrm{~N} \mathrm{~m}^{-2}$. As the wind forcing weakens, the form stress also weakens, which can happen either by reducing the magnitude of $\bar{\psi}_{2 x}^{\prime}$, the lower-layer time-averaged streamfunction, or by reducing its correlation with $h_{b}$. In these simulations both of these mechanisms occur. At zero wind, the form stress $h_{b} \bar{\psi}_{2 x}^{\prime}$ is not zero everywhere, but it is symmetric about the ridge (Fig. 6). Because of the symmetry, the form stress on one side of the ridge cancels out the form stress on the other side of the ridge, leading to no net form stress.

To relate the changes in form stress to the isopycnal slopes we must consider the connection between the form stress and the total eddy heat fluxes. The total eddy heat fluxes are given by $F_{1}\left\langle\overline{\psi_{1}^{\prime} \psi_{2 x}^{\prime}}\right\rangle$ which we can split up to be $F_{1}\left\langle\overline{\psi_{1}^{\prime} \psi_{2 x}^{\prime}}\right\rangle=F_{1}\left\langle\bar{\psi}_{1}^{\prime} \bar{\psi}_{2 x}^{\prime}\right\rangle+$ transients, where the first term on the right hand side, the standing eddy contribution, dominates over the transient term. So, if the magnitude of $\bar{\psi}_{2 x}^{\prime}$ decreases then the eddy heat fluxes in the meander will decrease. The correlation between these two terms could also decrease. In general, we see that $\bar{\psi}_{1}^{\prime}$ stays relatively symmetric about the ridge because the upper layer only sees the ridge through the lower layer (Fig. 3). As $\bar{\psi}_{2}^{\prime}$ becomes more symmetric about the ridge, the correlation between $\bar{\psi}_{1}^{\prime}$ and $\bar{\psi}_{2 x}^{\prime}$ will become smaller in the mean because the streamfunctions become more similar, leading to a cancellation of heat fluxes on either side of the ridge (Fig. 5b).

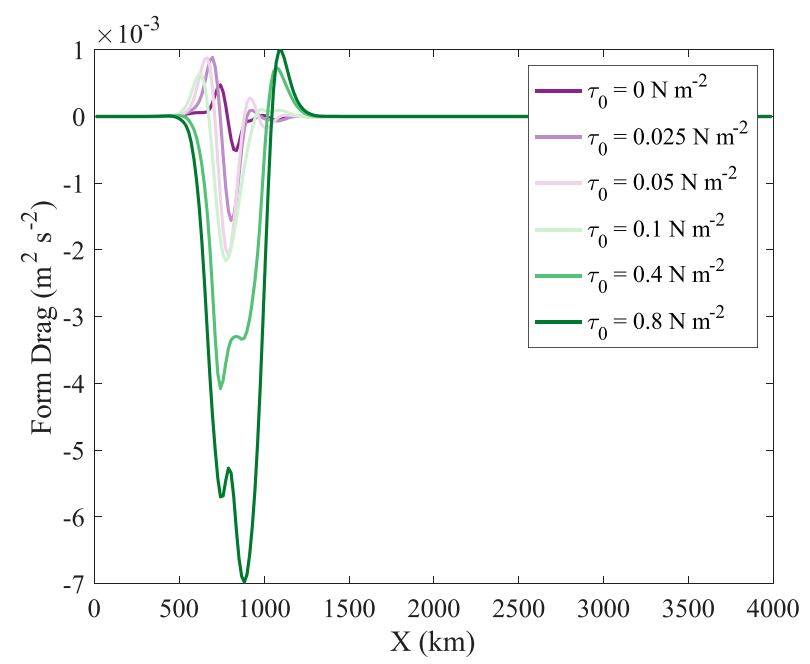

FIG. 6. Form drag along the channel for $\mathscr{H}_{0}=-2 \mathrm{~Sv}$. As the wind forcing decreases, the form drag becomes both weaker and more symmetric, but does not disappear altogether, leading to smaller net heat fluxes in the meander (Fig. 5). 
As the wind forcing decreases, the meander becomes smaller and more symmetric about the ridge and the ratio of the contribution to the fluxes from the meander and the rest of the domain decreases.

The result of the standing meander playing a smaller role in the heat fluxes is an increased sensitivity to wind forcing, but why do the thermal wind transports fan out as we observe (Fig. 4)? With strong wind stress, the cumulative eddy fluxes are concentrated in the meander, but as the wind forcing weakens the fluxes become important throughout the domain until the heat fluxes match the values for a flat-bottomed configuration (Fig. 5b). In this new regime, where the fluxes depend on the mean flow structure over the whole domain, the dynamics approach those of the flat-bottomed simulations where the eddy fluxes are uniform in $x$; thus, the thermal wind transport limits to the flat-bottomed transport as the winds weaken.

\section{c. No-wind limit}

Since the ridge simulations approach the flat-bottomed ones as the wind forcing weakens, it is important to understand the flat-bottomed simulations with no wind forcing. Intuitively, we would expect that the isopycnal slope change would be in proportion to the magnitude of the changes in the heat flux. We illustrate this using a linear approximation to our system, with no wind forcing and no eddies. The buoyancy forcing contribution to the upper-layer zonal mean potential vorticity equation can only be balanced by the $\kappa$ term, the potential vorticity diffusivity, in the steady state, that is,

$$
0=-\frac{f_{0} \mathscr{H}}{H_{1}}+\kappa \nabla^{2}\left\langle q_{1}\right\rangle
$$

Assuming that the potential vorticity is dominated by the stretching term, or $\left\langle q_{i}\right\rangle \approx \mp F_{i}\left(\left\langle\psi_{1}\right\rangle-\left\langle\psi_{2}\right\rangle\right)$, which is a good assumption considering the size of the domain, yields the upper-layer balance:

$$
\frac{f_{0} \kappa}{g^{\prime}} \nabla^{2}\left(\left\langle\psi_{1}\right\rangle-\left\langle\psi_{2}\right\rangle\right)=\mathscr{H}_{0} \sin (\pi y / L)
$$

We define $\psi_{\mathrm{BC}}=\left\langle\psi_{1}\right\rangle-\left\langle\psi_{2}\right\rangle$ and assume a solution of the form $\psi_{\mathrm{BC}}=\hat{\psi}_{\mathrm{BC}} \sin (\pi y / L)$. We plug this into Eq. (13) and find that $\hat{\psi}_{\mathrm{BC}}=g^{\prime} \mathscr{C}_{0} L^{2} /\left(f_{0} \kappa \pi^{2}\right)$, which tells us that the thermal wind transport, $\left\langle\overline{u_{1}}\right\rangle-\left\langle\overline{u_{2}}\right\rangle$, scales with $\mathscr{H}_{0}$, the magnitude of the heat fluxes. We compare this to the fully nonlinear channel model with and without topography in Fig. 7. With no wind stress and only heat flux, we see that the thermal wind transport follows our linear scaling until the overturning reaches $\mathscr{H}_{0}=-0.05 \mathrm{~Sv}$, at which point, eddies become important and the transport scales approximately like $\mathscr{H}_{0}^{1 / 5}$ (Fig. 7).

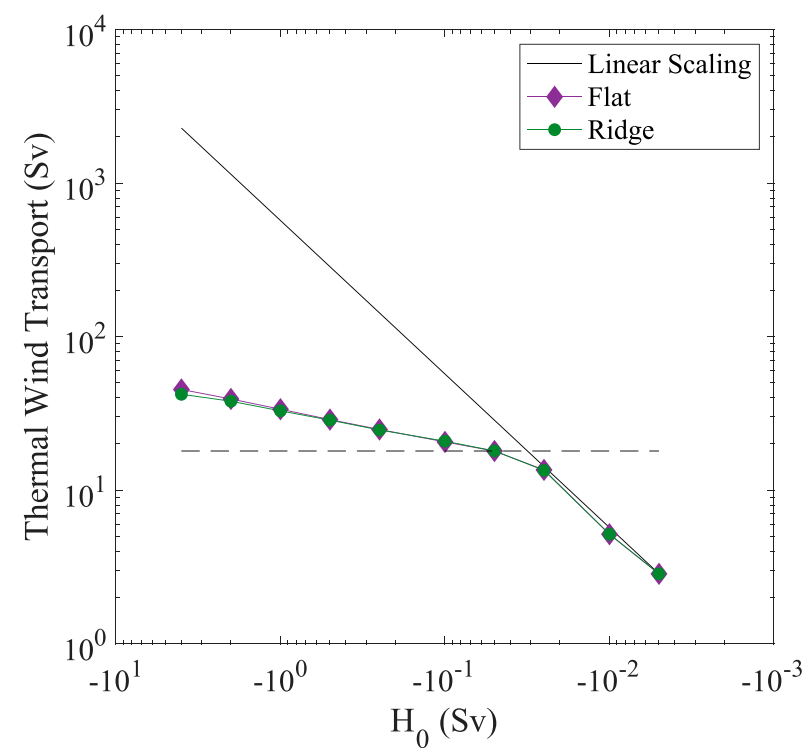

FIG. 7. Thermal wind transport vs residual overturning forced along interface for entire $\mathscr{H}_{0}<0$ range. The linear scaling developed is plotted against the simulated results that works well until the flow becomes eddying. The dashed line shows where the flow reaches its critical shear for instability following the Phillips problem. The thermal wind transport scales directly with the residual overturning and provides the no-wind limit to the thermal wind transport.

It is clear, however, as the forcing continues to grow, the thermal wind transport continues to grow with it, indicating that stronger heat fluxes do indeed generate steeper isopycnal slopes, a pattern that holds even with wind forcing and an eddying flow. Since the thermal wind transport must approach the values determined by the residual overturning when there is no wind forcing, the thermal wind transport cannot be saturated for every wind forcing in order to satisfy this limit.

\section{d. Connecting the limits}

Our results lead to the question of why must it be the case that the thermal wind transport decreases with increasing winds with topography but strengthens in the flat-bottomed case for a negative overturning. This pattern of decreasing thermal wind transport with an increase in wind is also seen, but not rationalized, in the channel models of Mak et al. (2018) and Munday and Zhai (2015). The thermal wind transport depends on the total eddy overturning and the wind forcing, but these two components vary with wind forcing differently. With stronger wind forcing, there is a smaller thermal wind transport because the meander grows and becomes asymmetric, isolating fluxes and increasing the eddy efficiency (Abernathey and Cessi 2014), leading to flatter isopycnal slopes (Fig. 8). There is 


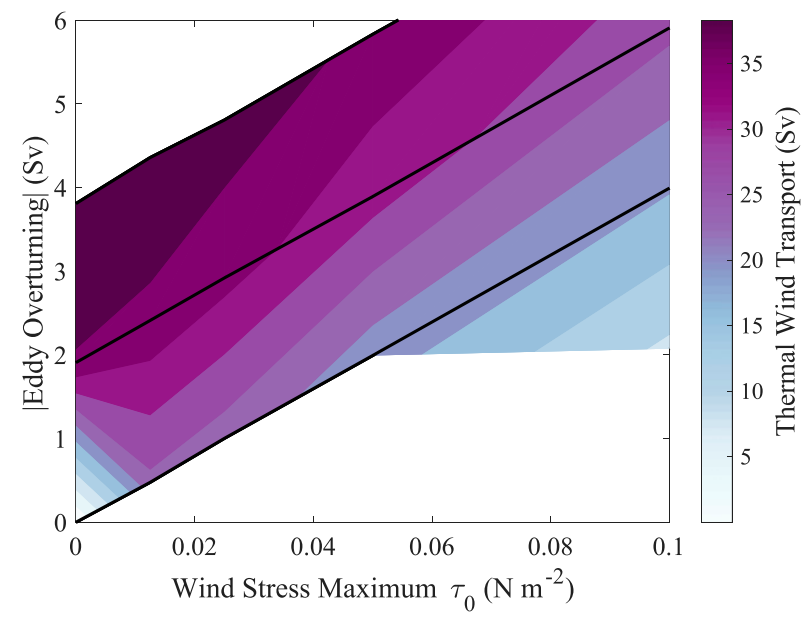

FIG. 8. The thermal wind transport vs the wind stress and total eddy overturning. The black lines correspond to a constant residual overturning. We see that for a given wind forcing, a stronger eddy overturning corresponds to a stronger thermal wind transport and for a given eddy overturning, a stronger wind forcing corresponds to a weaker thermal wind transport. However, as we increase the wind forcing along a line of constant overturning, the decreasing effect of the wind forcing overpowers the increasing effect of the eddy overturning, leading to smaller thermal wind transports as we increase the wind forcing for a negative residual overturning.

a singularity where the wind stress is zero because the total eddy overturning also goes to zero. With a stronger eddy overturning, there is a larger thermal wind transport because generally steeper isopycnal slopes lead to larger eddy fluxes and eddy overturning (Fig. 8). If we consider simulations where the residual overturning is constant, then when we increase the wind forcing, the eddy overturning increases as well, leading to opposing steepening and flattening of the isopycnals. We see that, of these two effects, the increase in eddy efficiency due to the meander wins over the need for more eddy activity (Fig. 8). Thus, the thermal wind transport decreases with an increasing wind forcing when there is a negative overturning.

\section{Discussion and conclusions}

The thermal wind transport in the idealized Southern Ocean-like channel model employed here is sensitive to changes in wind forcing at realistic wind stresses, between 0.1 and $0.2 \mathrm{Nm}^{-2}$, albeit in this idealized framework it is hard to make quantitative comparisons. At wind stresses below $\tau_{0}=0.4 \mathrm{~N} \mathrm{~m}^{-2}$, the transport either increases or decreases with the wind depending on the residual overturning because the simulations limits to those with a flat bottom as the winds weaken (Fig. 9). The convergence occurs because for weaker wind stress the amplitude of the standing meander decreases and thus the system becomes more zonally symmetric as in a flat-bottomed channel. Our results and arguments show the limitations of eddy saturation as a theory even in an idealized configuration when there is a residual overturning.

Our results differ from previous studies, in part because we isolate the positive and negative overturning cells. This is different than Hogg (2010), where there are both negative and positive buoyancy fluxes. Hogg (2010) also lacks a proper residual overturning where there is a meridional circulation at depth, but instead has all the meridional transport in a mixed layer, and the wind forcing is also happening in different location as the buoyancy forcing. Hallberg and Gnanadesikan (2001) also only considers a positive overturning, so they cannot see the different behavior with the negative overturning. It is possible that our results would be consistent with these past studies if we considered similar forcing regimes and could look at each overturning cell individually.

This study does not directly address why eddy saturation exists in these simplified models, but hopefully, by extending the work of Abernathey and Cessi (2014) and Nadeau and Ferrari (2015), it provides insight into the relative importance of the meander. Working to understand the meander spinup process also merits further study. It is not clear why the standing meander is so much more efficient at flattening isopycnal slopes than the wind-driven process of increasing the slopes. Mak et al. (2018) encapsulate the eddy efficiency in their $\alpha$ parameter. In our configuration this $\alpha$ must change if the transport changes; there are no other parameters that could vary to change the transport, though it is unclear how or why the eddy efficiency changes.

One other open question is what sets the value of wind stress where saturation begins. The saturation does not depend on the relative strength of the wind-driven overturning, but the absolute wind stress. It seems likely to depend on when the isolation of the fluxes to the ridge area begins. Understanding the isolation process has serious implications for predicting how the Southern Ocean thermal wind transport and residual overturning will respond to changes in the wind.

The geometry is simpler than the real Southern Ocean, but the dominant feature of a blocking ridge is present. The ridge is too small compared to real topographic features (Thompson and Naveira Garabato 2014), so the simulations have been repeated with a 2000-m-tall ridge, and the saturated thermal wind transport value is the same. The main difference is that the saturation breaks down closer to $\tau_{0}=0.3 \mathrm{~N} \mathrm{~m}^{-2}$ versus $\tau_{0}=0.4 \mathrm{~N} \mathrm{~m}^{-2}$ for a $1000-\mathrm{m}$-tall ridge, but the main results still hold. Another potential limit to our 


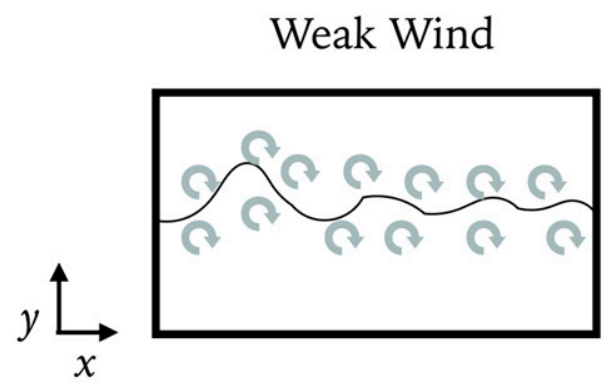
Strong Wind

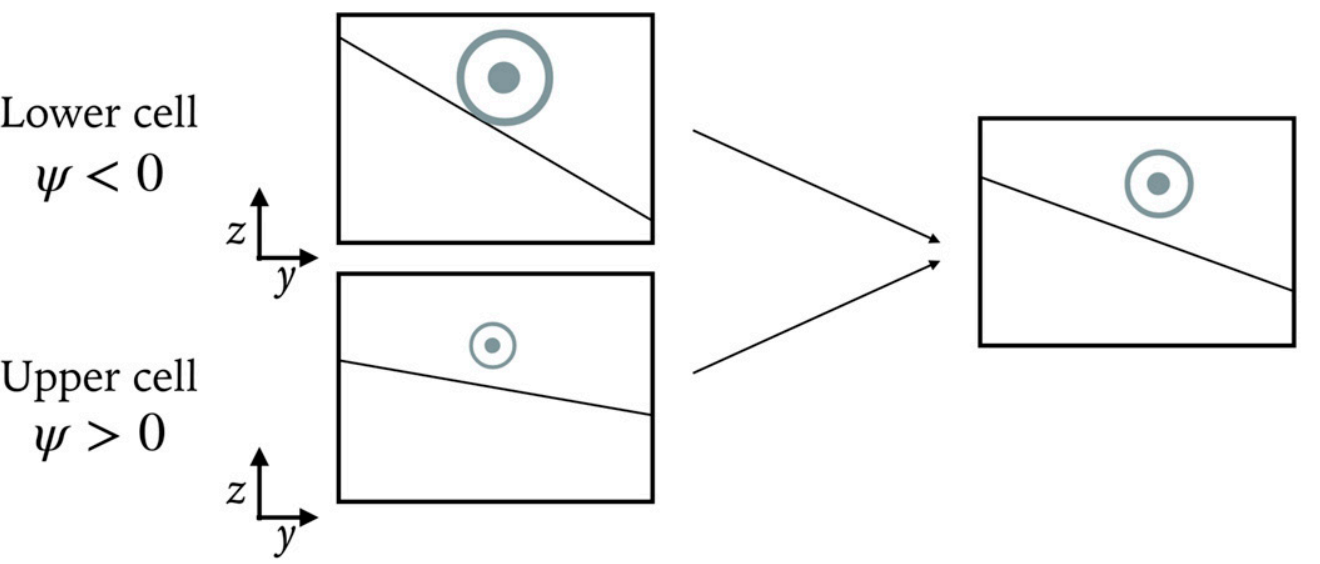

FIG. 9. A concluding schematic. When there is a weak wind forcing, the strength of the thermal wind transport is dependent on the sign of the overturning, the meander is symmetric about the ridge, and the eddy activity is spread throughout the domain. When the wind forcing is strong the eddy activity is localized to the meander region, the meander is asymmetric, and the thermal wind transport no longer depends on the overturning.

result is the use of two-layer quasigeostrophic simulations. The simplified dynamics could lead to potential problems due to the lack of outcrops as seen in the real Southern Ocean, so to this end, we will be carrying out analogous investigations in primitive models using MITgcm simulations with the same geometry; preliminary experiments are broadly consistent with the results presented in this study. Another approach to address this problem would be to consider a three-layer quasigeostrophic model to extend our result with both a positive and a negative cell at the same time. It is unclear how these results would translate into primitive equation channel models, especially whether eddy saturation would still kick in only at unrealistically strong wind stresses.

Our system is simple in order to fully explore the parameter space of baroclinic instability. The result from this study is useful for considering more complicated models and provides a framework to analyze changes in thermal wind transport with wind based on the magnitude of the residual overturning. We show that both topography and residual overturning are vital components for any theory of saturation. It is possible that saturation seen in various models and the real Antarctic Circumpolar Current with two overturning cells is actually due to the wind forcing changing the thermal wind transport in opposite directions in each overturning cell. If we connect these isopycnal slope patterns to outcrop positions in the real ocean, then we could also provide insight into the changes of the residual overturning with wind forcings with implications for fluxes of heat and carbon.

Acknowledgments. MKY and RF acknowledge support through NSF Awards OCE-1536515 and AGS1835576. MKY acknowledges funding from NDSEG. GRF was supported by NSF OCE-1459702. We are very grateful for conversations with David Marshall, Andrew Stewart, and two anonymous reviewers that greatly improved the manuscript. The code for running the model is found at https://github.com/mkyoungs/ JPO-QG-Channel.

\section{APPENDIX}

\section{Derivation of Equations}

We feel it is informative to systematically trace the steps in developing the equations starting from the 
two-layer shallow water system. We will incorporate the residual flow transformation and introduce the appropriate potential vorticity-like quantity, then examine the zonal mean momentum and mass equations. Finally, we comment on the quasigeostrophic approximation.

We start with

$$
\begin{aligned}
\frac{\partial}{\partial t} \mathbf{u}_{i}+\hat{\mathbf{z}} \times\left(\zeta_{i}+f_{0}+\beta y\right) \mathbf{u}_{i} & =-\nabla\left(p_{i}+\frac{1}{2}\left|\mathbf{u}_{i}\right|^{2}\right)+\mathbf{T}_{i}, \\
\frac{\partial}{\partial t} h_{i}+\nabla \cdot\left(\mathbf{u}_{i} h_{i}\right) & = \pm \mathscr{H}, \quad \text { and } \\
p_{1}-p_{2} & =g^{\prime} h_{1},
\end{aligned}
$$

where $\mathbf{T}$ represents the nonconservative forces of wind stress and friction and $\zeta_{i}$ is the relative vorticity. The upper and lower signs apply for the water above $(i=1)$ and below $(i=2)$ the thermocline. The crossinterface mass transfer $\mathscr{H}$ can be thought of as buoyancy forcing and is responsible for a residual mean circulation.

The mass equations are used to define rotational and divergent transports (Kushner and Held 1999)

$$
\mathbf{u}_{i} \frac{h_{i}}{H_{i}}=\hat{\mathbf{z}} \times \nabla \psi_{i}+\mathbf{u}_{i}^{*},
$$

with $\mathbf{u}^{*}$ representing the residual flow. The relationship to $\mathscr{H}$ becomes obvious since the mass equations are now linear:

$$
\frac{\partial}{\partial t} h_{i}+H_{i} \nabla \cdot \mathbf{u}_{i}^{*}= \pm \mathscr{H} .
$$

The changes of sea surface elevation, while responsible for the upper-layer horizontal pressure gradients, can be neglected in the mass balance; therefore $h_{1}+h_{2}$ is constant. This implies that the barotropic residual flow is nondivergent

$$
\nabla \cdot\left(H_{1} \mathbf{u}_{1}^{*}+H_{2} \mathbf{u}_{2}^{*}\right)=0 .
$$

Therefore, we only need the upper-layer thickness equation

$$
\frac{\partial}{\partial t} h_{1}+H_{1} \nabla \cdot \mathbf{u}_{1}^{*}=\mathscr{H} .
$$

Note that the definition of the residual circulation is incomplete: we have replaced two fields giving the mass transport by three. We are free, therefore, to specify the boundary conditions: the normal flow vanishes for both $\psi$ and $\mathbf{u}^{*}$. Note also that the residual vertical velocity $w^{*}= \pm \nabla \cdot \mathbf{u}_{i}^{*} / H_{i}$ is the same just above and below the interface, unlike $w$, which includes flow along a sloping interface.

The momentum equations will be rewritten with the absolute vorticity replaced by the (nearly) quasigeostrophic potential vorticity

$$
q_{i}=\nabla^{2} \psi_{i}+\beta y \mp \frac{f_{0}}{H_{i}}\left(h_{1}-H_{1}\right)+\delta_{i 2} \frac{f_{0}}{H_{i}} h_{b}
$$

and $p_{i}=f_{0} \psi_{i}+p_{i}^{*}$. The result,

$$
\begin{aligned}
\frac{\partial}{\partial t} \mathbf{u}_{i} & +\hat{\mathbf{z}} \times f_{0}\left(1+\frac{h_{i}-H_{i}}{H_{i}}\right) \mathbf{u}_{i}+\hat{\mathbf{z}} \times q_{i} \mathbf{u}_{i} \\
& =-\nabla\left(p_{i}^{*}+\frac{1}{2}|\mathbf{u}|^{2}\right),
\end{aligned}
$$

becomes

$$
\frac{\partial}{\partial t} \mathbf{u}_{i}+\hat{\mathbf{z}} \times f_{0} \mathbf{u}_{i}^{*}+\hat{\mathbf{z}} \times q_{i} \mathbf{u}_{i}=-\nabla\left(p_{i}^{*}+\frac{1}{2}|\mathbf{u}|^{2}\right),
$$

with $f_{0}\left(\psi_{1}-\psi_{2}\right)+p_{1}^{*}-p_{2}^{*}=g^{\prime}\left(h_{1}-H_{1}\right)$. The equation for the evolution of $q_{i}$ follows easily by taking the curl of the momentum equation and adding $\mp f_{0} / H_{i}$ times the mass equation:

$$
\frac{\partial}{\partial t} q_{i}+\nabla \cdot\left(q_{i} \mathbf{u}_{i}\right)=\nabla \times \mathbf{T}_{i}-\frac{f_{0}}{H_{i}} \mathscr{H} .
$$

Finally, the zonally averaged zonal momentum and thickness equations are

$$
\begin{aligned}
\frac{\partial}{\partial t}\left\langle u_{i}\right\rangle-f_{0}\left\langle v_{i}^{*}\right\rangle-\left\langle v_{i} q_{i}\right\rangle & =\left\langle T_{i}^{x}\right\rangle, \\
\frac{\partial}{\partial t}\left\langle h_{1}\right\rangle+H_{1} \frac{\partial}{\partial y}\left\langle v_{1}^{*}\right\rangle & =\langle\mathscr{H}\rangle .
\end{aligned}
$$

So far, Eqs. (A1)-(A5) are exact. We now approximate by 1$)$ replacing $1+\left(h_{i}-H_{i}\right) / H_{i}$ by 1,2$)$ dropping $\mathbf{u}^{*}$ terms when combined with the lower order in Rossby number rotational flow to get $\mathbf{u} \simeq \hat{\mathbf{z}} \times \nabla \psi$, and 3) dropping the $p^{*}$ in the hydrostatic equation so that $f_{0}\left(\psi_{1}-\psi_{2}\right)=g^{\prime}\left(h_{1}-H_{1}\right)$. We then have the quasigeostrophic equations

$$
\frac{\partial}{\partial t} q_{i}+\mathbf{u}_{i} \cdot \nabla q_{i}=\nabla \times \mathbf{T}_{i}-\frac{f_{0}}{H_{i}} \mathscr{H},
$$

with

$$
\begin{aligned}
& \mathbf{u}_{i}=\hat{\mathbf{z}} \times \psi_{i} \quad \text { and } \\
& q_{i}=\nabla^{2} \psi_{i} \mp F_{i}\left(\psi_{1}-\psi_{2}\right)+\beta y+\delta_{i 2} \frac{f_{0}}{H_{2}} h_{b},
\end{aligned}
$$


where $F_{i}$ is defined in section 2 . These will be solved for the zonally varying part

$$
\begin{aligned}
\frac{\partial}{\partial t} q_{i}^{\prime} & +\left\langle u_{i}\right\rangle \frac{\partial}{\partial x} q_{i}^{\prime}+v_{i}^{\prime} \frac{\partial}{\partial y}\left\langle q_{i}\right\rangle+\frac{\partial}{\partial x}\left(u_{i}^{\prime} q_{i}^{\prime}\right)+\frac{\partial}{\partial y}\left(v_{i}^{\prime} q_{i}^{\prime}-\left\langle v_{i}^{\prime} q_{i}^{\prime}\right\rangle\right) \\
& =\nabla \times \mathbf{T}_{i}^{\prime}, \\
q_{i}^{\prime} & =\nabla^{2} \psi_{i}^{\prime} \mp F_{i}\left(\psi_{1}^{\prime}-\psi_{2}^{\prime}\right)+\delta_{i 2} \frac{f_{0}}{H_{2}} h_{b} .
\end{aligned}
$$

Consistent with quasi-geostrophy, we can use the zonal mean thermal wind equation,

$$
f_{0}\left(\left\langle u_{1}\right\rangle-\left\langle u_{2}\right\rangle\right)=-g^{\prime} \frac{\partial}{\partial y}\left(h_{1}-H_{1}\right)
$$

to eliminate the time-derivative terms from Eqs. (A2) and (A3) and derive an omega equation

$$
\begin{aligned}
\left(\frac{\partial^{2}}{\partial y^{2}}-F_{1}-F_{2}\right)\left\langle v_{1}^{*}\right\rangle= & \frac{f_{0}}{g^{\prime} H_{1}}\left(\left\langle v_{1}^{\prime} q_{1}^{\prime}\right\rangle-\left\langle v_{2}^{\prime} q_{2}^{\prime}\right\rangle+\left\langle T_{1}^{x}\right\rangle\right. \\
& \left.-\left\langle T_{2}^{x}\right\rangle\right)+\frac{1}{H_{1}} \frac{\partial}{\partial y}\langle\mathscr{H}\rangle .
\end{aligned}
$$

\section{REFERENCES}

Abernathey, R. P., and P. Cessi, 2014: Topographic enhancement of eddy efficiency in baroclinic equilibration. J. Phys. Oceanogr., 44, 2107-2126, https://doi.org/10.1175/ JPO-D-14-0014.1.

Barthel, A., A. McC. Hogg, S. Waterman, and S. Keating, 2017: Jet-topography interactions affect energy pathways to the deep Southern Ocean. J. Phys. Oceanogr., 47, 1799-1816, https://doi.org/10.1175/JPO-D-16-0220.1.

Bishop, S. P., P. R. Gent, F. O. Bryan, A. F. Thompson, M. C. Long, and R. P. Abernathey, 2016: Southern Ocean overturning compensation in an eddy-resolving climate simulation. J. Phys. Oceanogr., 46, 1575-1592, https://doi.org/ 10.1175/JPO-D-15-0177.1.

Chelton, D. B., R. A. DeSzoeke, M. G. Schlax, K. El Naggar, and N. Siwertz, 1998: Geographical variability of the first baroclinic Rossby radius of deformation. J. Phys. Oceanogr., 28, 433-460, https://doi.org/10.1175/1520-0485(1998)028<0433: GVOTFB $>2.0 . \mathrm{CO} ; 2$.

Constantinou, N. C., 2018: A barotropic model of eddy saturation. J. Phys. Oceanogr., 48, 397-411, https://doi.org/10.1175/ JPO-D-17-0182.1.

— , and W. R. Young, 2017: Beta-plane turbulence above monoscale topography. J. Fluid Mech., 827, 415-447, https://doi.org/10.1017/ jfm.2017.482.

Danabasoglu, G., J. C. Mcwilliams, and P. R. Gent, 1994: The role of mesoscale tracer transports in the global ocean circulation. Science, 264, 1123-1126, https://doi.org/10.1126/ SCIENCE.264.5162.1123.

Farneti, R., and Coauthors, 2015: An assessment of Antarctic Circumpolar Current and Southern Ocean meridional overturning circulation during 1958-2007 in a suite of interannual
CORE-II simulations. Ocean Modell., 93, 84-120, https:// doi.org/10.1016/j.ocemod.2015.07.009.

Flierl, G. R., and J. Pedlosky, 2007: The nonlinear dynamics of time-dependent subcritical baroclinic currents. J. Phys. Oceanogr., 37, 1001-1021, https://doi.org/10.1175/JPO3034.1.

Gnanadesikan, A., 1999: A simple predictive model for the structure of the oceanic pycnocline. Science, 283, 2077-2079, https://doi.org/10.1126/science.283.5410.2077.

_- , and R. W. Hallberg, 2000: On the relationship of the circumpolar current to Southern Hemisphere winds in coarse-resolution ocean models. J. Phys. Oceanogr., 30 , 2013-2034, https://doi.org/10.1175/1520-0485(2000)030<2013: OTROTC $>2.0 . \mathrm{CO} ; 2$.

Hallberg, R. W., and A. Gnanadesikan, 2001: An exploration of the role of transient eddies in determining the transport of a zonally reentrant current. J. Phys. Oceanogr., 31, 3312-3330, https://doi.org/10.1175/1520-0485(2001)031<3312:AEOTRO> 2.0.CO;2.

Hogg, A. McC., 2010: An Antarctic Circumpolar Current driven by surface buoyancy forcing. Geophys. Res. Lett., 37, L23601, https://doi.org/10.1029/2010GL044777.

—, M. P. Meredith, D. P. Chambers, E. P. Abrahamsen, C. W. Hughes, and A. K. Morrison, 2015: Recent trends in the Southern Ocean eddy field. J. Geophys. Res. Oceans, 120, 257 267, https://doi.org/10.1002/2014JC010470.

Howard, E., A. McC. Hogg, S. Waterman, and D. P. Marshall, 2015: The injection of zonal momentum by buoyancy forcing in a Southern Ocean model. J. Phys. Oceanogr., 45, 259-271, https://doi.org/10.1175/JPO-D-14-0098.1.

Johnson, G. C., and H. L. Bryden, 1989: On the size of the Antarctic Circumpolar Current. Deep-Sea Res., 36, 39-53, https:// doi.org/10.1016/0198-0149(89)90017-4.

Karsten, R. H., and J. Marshall, 2002: Testing theories of the vertical stratification of the Antarctic Circumpolar Current against observations. Dyn. Atmos. Oceans, 36, 233-246, https://doi.org/10.1016/S0377-0265(02)00031-3.

Kushner, P. J., and I. M. Held, 1999: Potential vorticity thickness fluxes and wave-mean flow interaction. J. Atmos. Sci., 56, 948-958, https://doi.org/10.1175/1520-0469(1999)056<0948: PVTFAW $>2.0 . \mathrm{CO} ; 2$.

Lin, X., X. Zhai, Z. Wang, and D. R. Munday, 2018: Mean, variability, and trend of Southern Ocean wind stress: Role of wind fluctuations. J. Climate, 31, 3557-3573, https://doi.org/10.1175/ JCLI-D-17-0481.1.

Lumpkin, R., and K. Speer, 2007: Global ocean meridional overturning. J. Phys. Oceanogr., 37, 2550-2562, https://doi.org/ 10.1175/JPO3130.1.

MacCready, P., and P. B. Rhines, 2001: Meridional transport across a zonal channel: Topographic localization. J. Phys. Oceanogr., 31, 1427-1439, https://doi.org/10.1175/1520-0485(2001)031<1427: MTAAZC $>2.0 . \mathrm{CO} ; 2$.

Mak, J., D. P. Marshall, J. R. Maddison, and S. D. Bachman, 2017: Emergent eddy saturation from an energy constrained eddy parameterisation. Ocean Modell., 112, 125-138, https:// doi.org/10.1016/j.ocemod.2017.02.007.

— J. R. Maddison, D. P. Marshall, and D. R. Munday, 2018: Implementation of a geometrically informed and energetically constrained mesoscale eddy parameterization in an ocean circulation model. J. Phys. Oceanogr., 48, 2363-2382, https://doi.org/10.1175/JPO-D-18-0017.1.

Marshall, D. P., 1997: Subduction of water masses in an eddying ocean. J. Mar. Res., 55, 201-222, https://doi.org/10.1357/ 0022240973224373. 
Marshall, G. J., 2003: Trends in the southern annular mode from observations and reanalyses. J. Climate, 16, 4134-4143, https://doi.org/10.1175/1520-0442(2003)016<4134:TITSAM> 2.0.CO;2.

Marshall, J., and T. Radko, 2003: Residual-mean solutions for the Antarctic Circumpolar Current and its associated overturning circulation. J. Phys. Oceanogr., 33, 2341-2354, https://doi.org/ 10.1175/1520-0485(2003)033<2341:RSFTAC $>2.0$.CO;2.

— circulation through Southern Ocean upwelling. Nat. Geosci., 5, 171-180, https://doi.org/10.1038/ngeo1391.

Marshall, D. P., M. H. Ambaum, J. R. Maddison, D. R. Munday, and L. Novak, 2017: Eddy saturation and frictional control of the Antarctic Circumpolar Current. Geophys. Res. Lett., 44, 286-292, https://doi.org/10.1002/2016GL071702.

McWilliams, J. C., W. R. Holland, and J. H. Chow, 1978: A description of numerical Antarctic Circumpolar Currents. Dyn. Atmos. Oceans, 2, 213-291, https://doi.org/10.1016/ 0377-0265(78)90018-0.

Meredith, M. P., and A. McC. Hogg, 2006: Circumpolar response of Southern Ocean eddy activity to a change in the Southern Annular Mode. Geophys. Res. Lett., 33, L16608, https:// doi.org/10.1029/2006GL026499.

Morrison, A. K., A. M. C. Hogg, and M. L. Ward, 2011: Sensitivity of the Southern Ocean overturning circulation to surface buoyancy forcing. Geophys. Res. Lett., 38, L14602, https:// doi.org/10.1029/2011GL048031.

Munday, D. R., and X. Zhai, 2015: Sensitivity of Southern Ocean circulation to wind stress changes: Role of relative wind stress. Ocean Modell., 95, 15-24, https://doi.org/10.1016/ j.ocemod.2015.08.004.

- H. L. Johnson, and D. P. Marshall, 2013: Eddy saturation of equilibrated circumpolar currents. J. Phys. Oceanogr., $\mathbf{4 3}$, 507-532, https://doi.org/10.1175/JPO-D-12-095.1.

$\ldots,-$, and -2015 : The role of ocean gateways in the dynamics and sensitivity to wind stress of the early Antarctic Circumpolar Current. Paleoceanography, 30, 284-302, https:// doi.org/10.1002/2014PA002675.
Munk, W. H., and E. Palmén, 1951: Note on the dynamics of the Antarctic Circumpolar Current. Tellus, 3, 53-55, https:// doi.org/10.3402/tellusa.v3i1.8609.

Nadeau, L.-P., and R. Ferrari, 2015: The role of closed gyres in setting the zonal transport of the Antarctic Circumpolar Current. J. Phys. Oceanogr., 45, 1491-1509, https://doi.org/ 10.1175/JPO-D-14-0173.1.

Nikurashin, M., and G. K. Vallis, 2011: A theory of deep stratification and overturning circulation in the ocean. J. Phys. Oceanogr., 41, 485-502, https://doi.org/10.1175/ 2010JPO4529.1.

Pedlosky, J., 1987: Geophysical Fluid Dynamics. Springer, 710 pp.

Plumb, R. A., and R. Ferrari, 2005: Transformed Eulerian-mean theory. Part I: nonquasigeostrophic theory for eddies on a zonal-mean flow. J. Phys. Oceanogr., 35, 165-174, https:// doi.org/10.1175/JPO-2669.1.

Shakespeare, C. J., and A. M. Hogg, 2012: An analytical model of the response of the meridional overturning circulation to changes in wind and buoyancy forcing. J. Phys. Oceanogr., 42, 1270-1287, https://doi.org/10.1175/JPO-D-11-0198.1.

Shepherd, T. G., 1983: Mean motions induced by baroclinic instability in a jet. Geophys. Astrophys. Fluid Dyn., 27, 35-72, https://doi.org/10.1080/03091928308210120.

Straub, D. N., 1993: On the transport and angular momentum balance of channel models of the Antarctic Circumpolar Current. J. Phys. Oceanogr., 23, 776-782, https://doi.org/10.1175/ 1520-0485(1993)023<0776:OTTAAM $>2.0$. CO;2.

Thompson, A. F., and A. C. Naveira Garabato, 2014: Equilibration of the Antarctic Circumpolar Current by standing meanders. J. Phys. Oceanogr., 44, 1811-1828, https://doi.org/10.1175/ JPO-D-13-0163.1.

Thompson, D. W. J., and S. Solomon, 2002: Interpretation of recent Southern Hemisphere climate change. Science, 296, 895-899, https://doi.org/10.1126/science.1069270.

Youngs, M. K., A. F. Thompson, A. Lazar, and K. J. Richards, 2017: ACC meanders, energy transfer, and mixed barotropicbaroclinic instability. J. Phys. Oceanogr., 47, 1291-1305, https:// doi.org/10.1175/JPO-D-16-0160.1. 